\title{
Performance evaluation of ad-hoc based aerial monitoring system
}

\author{
Salih M. Al-Qaraawi ${ }^{1}$, Aymen D. Salman ${ }^{2}$, Majida S. Ibrahim ${ }^{3 *}$ \\ ${ }^{1,2,3}$ Computer Engineering Dept., University of Technology, Baghdad, Iraq
}

\begin{abstract}
Recently there is a huge interest in designing and implementing systems that can be used in surveillance and emergency situations. These systems are designed and implemented using two main technologies that are: Mobile Adhoc Networks (MANETs) and the Unmanned Aerial Vehicles (UAVs). MANETs with its unique characteristics of rapid deployment, self-organization and cost effectivenes had made it a popular topic for designers and developers to design and implement such systems. In this paper, a prototype system was designed and implemented using MANETs and UAVs; this system can be developed to be used as an aerial monitoring system in surveillance and security issues, the system was used to record and send a real-time video from source to destination node over a multihop path. This system was first implemented and tested using testbed method, then it was simulated using network simulator (NS-3) with two case studies to evaluate the performance of the system using two routing protocols (Ad-hoc On-Demand Destance Vector AODV[1] and Optimised Link State Routing OLSR [2]). The evaluating metrics used here are; delay, average jitter, packet loss ratio (PLR) and packet delivery factor (PDF) against variable number of nodes. The optained results of the test bed method showed the configuration parameters and self-organization characteristics of MANET, the results obtained from the simulation platform illustrated that the OLSR had outperformed the AODV protocol in dense networks and the optimum number of nodes needed to cover the simulation area were 90 nodes.
\end{abstract}

Keywords: $\quad$ MANETs, UAVs, Aerial monitoring, OLSR and AODV, Performance evaluation

Corresponding Author:

Majida S. Ibrahim

Departement, Computer Engineering

University of Technology

Baghdad, Iraq

E-mail: majiwsaud@gmail.com

\section{Introduction}

In surveillance and critical issues applications, where the use of normal-infrastructure networks are infeasible, ad-hoc networks are the best replacement, such applications as military where it is required to locate targets deep in battlefield [3], disaster management, emergency situations as in search operations were the use of Quad-Copters with ad-hoc mode leads to gain more coverage area [4].

MANETs are a collection of homogenous or heterogeneous nodes that are communicating with each other via a wireless interface. As shown in Figure 1.

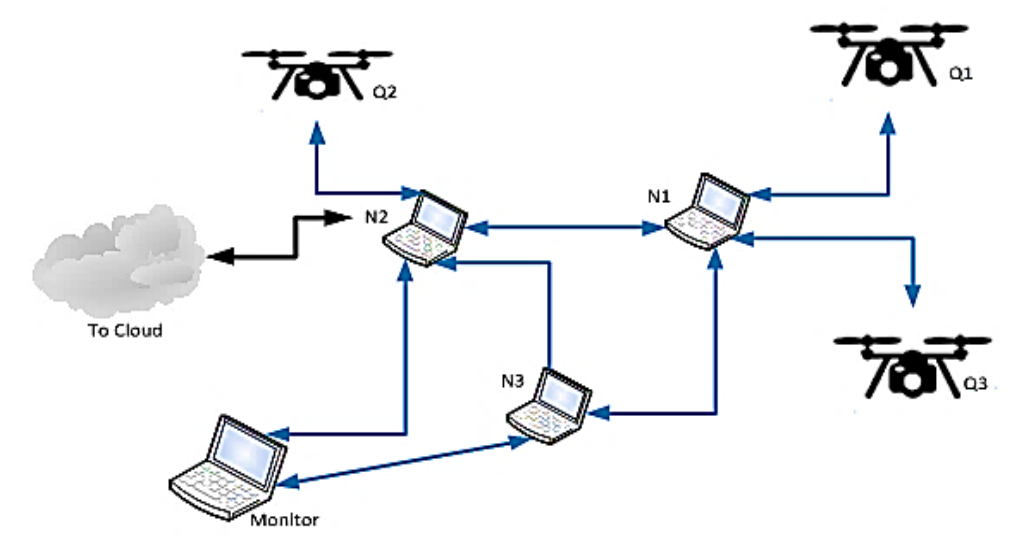

Figure 1. Ad-hoc network 
MANETs are characterized by the frequent and unpredictable changes of topology which make the operation of finding and establishing a path between any two communicating nodes is a real challenge.

The researchers in this field had developed many routing algorithms to maintain the connection from any source to any destination in the network. Routing protocols are classified into three main categories [5]: proactive or table-driven routing protocols, reactive or on-demand routing protocols, hybrid routing protocols. Quadcopters are a certain type of UAVs that had been increasingly interested due to its flight stability and maneuverability. Quadcopters had been used in emergency and rescue systems in hazardous places such as; big woods, mountains, nuclear stations, aerial monitoring, etc. in such applications where the interference of human teams are dangerous and costly.

\section{Related studies}

Recently, there is a big interest in providing means of monitoring for surveillance, emergency and disasters situations; UAVs, ad-hoc networks, and visualization tools are some technologies that serve the early mentioned applications.

Some researchers had introduced some scientific papers and proposed systems in the field of using Quadcopters along with an Ad-hoc mechanism to develop systems that can be used in various applications; some related studies are listed below:

Yixin Gu Et Al., [6] designed a system that uses the wireless interface mounted on the Quadcopter to implement an ad-hoc network used in monitoring and fighting big wood fires. The designed systems were used to transfer real-time video to enable the activities of rescue systems.

Carlos Cambra et al., [4] used the UAVs in designing an emergency rescue system that use Quadcopters to receive signals from mountaineer's smartphones and locate them in search operations. Quadcopters were used to expand the searching area in disasters such as; earth quick, big fires or any situation where the use of normal wireless communication is impossible and costly.

Omer Shrit et al., [7] used ad-hoc networks to combine and synchronize a swarm of Quadcopters to be used in surveillance and remote monitoring of big crowds.

Another new usage of Drones and ad-hoc network technologies in combining communicating nodes on the ground using an access point mounted on the Quadcopter, the system proposed in [8] by H. Ullah et al., was used in emergency and disaster management situations.

IDan Radu et al., [9] proposed a system that depends on technologies used in an emergency that are: Internet of Things and mobile ad-hoc networks. The proposed system used Multi Path-Optimized Link State Routing (MP-OLSR) protocol [10] over Flying Ad-hoc Networks FANETs to collect data about big fires that leads to saving people.

\section{Proposed system}

The University of Technology is one of the well-known Iraqi universities with the big area of $(1050 * 780 \mathrm{~m})$. It contains 14-scientific departments, 3-search centers, 2-scientific centers, 4- service centers and six search units, in addition to other entertainment units. The number of its academic stuff is more than 1380, undergraduate students are about 6105 and postgraduate students are 386 besides the visitors [11].

Such a big campus with that tremendous number of people are getting in/out of the campus daily to make the monitoring operation of it exits in rush hours and controll the security issues is a real challenge. The proposed prototype system can be developed to be used in aerial monitoring for the three-main exits of the campus.

The ad-hoc network mode was selected along with Quadcopters to design a practical system to be used in the monitoring process for large areas with no system-implementation and time limitations.

\section{System implementation and testing}

The proposed prototype system in this paper was implemented using Raspberry pi 3 (Rpi3), pi-camera (as the source of real-time video), a laptop (as the monitor or destination node) and the Quadcopter (as the mobile device in the system). The pi-camera was connected to the Rpi3 and was used to record the real-time video which was then sent to the monitor node over a multihop path. 
This system was tested using the testbed method to test the configuration of ad-hoc mode parameters using "iwconfig" command and the self-organization mechnisim using "traceroute" command. Figure 2. showed the implemented prototype system.

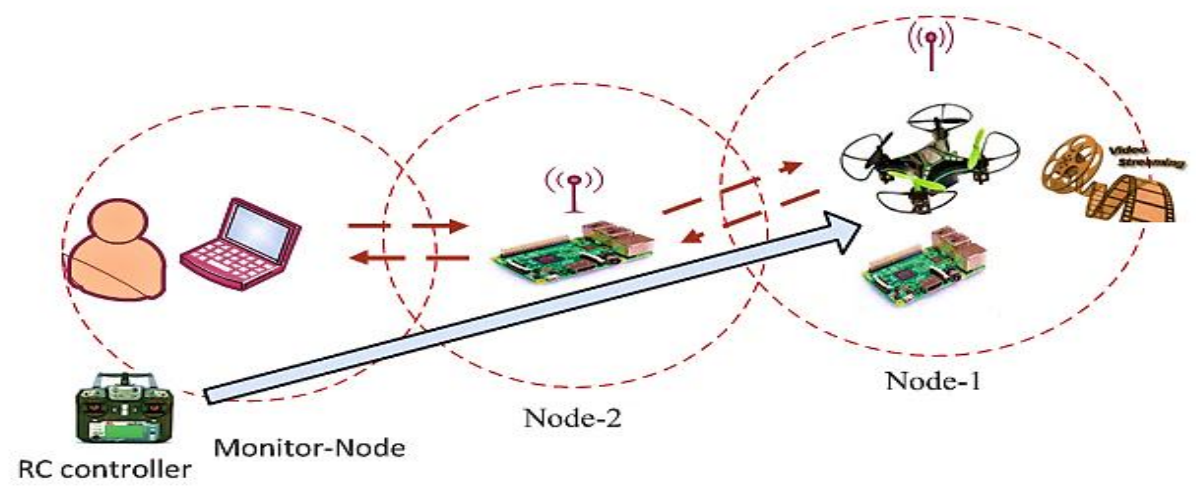

Figure 2. Proposed Prototype system

Figure 3 showed the result of "iwconfig" command execution, where the ad-hoc mode parameters are shown.

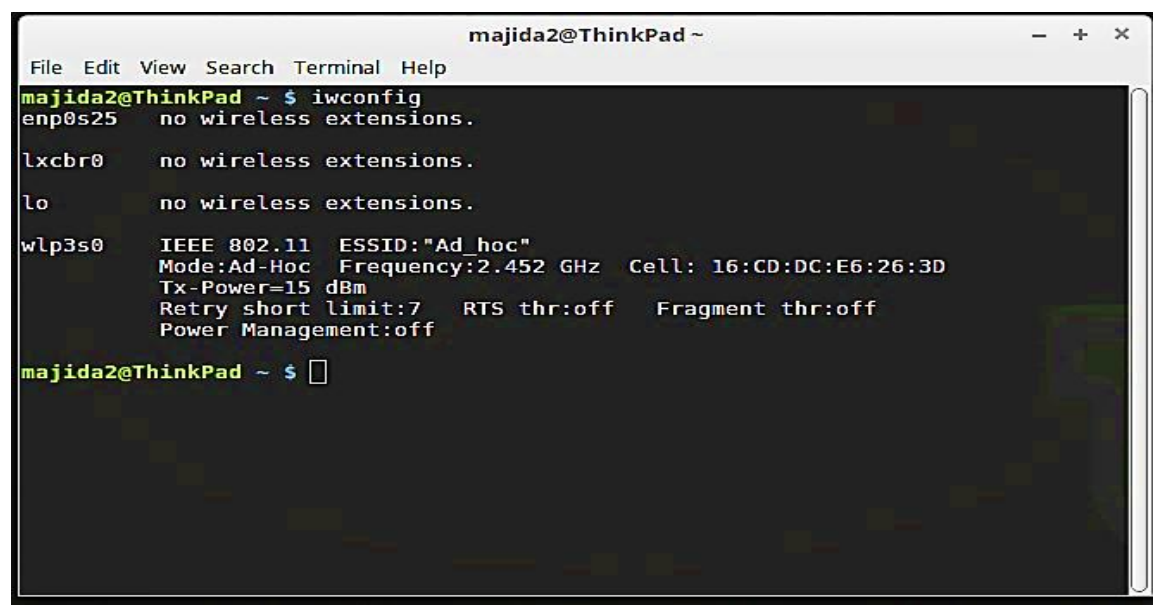

Figure 3. "Iwconfig" command execution

Figures 4 showed the results of one hop traceroute where the the source and destination nodes are within the range of each others.

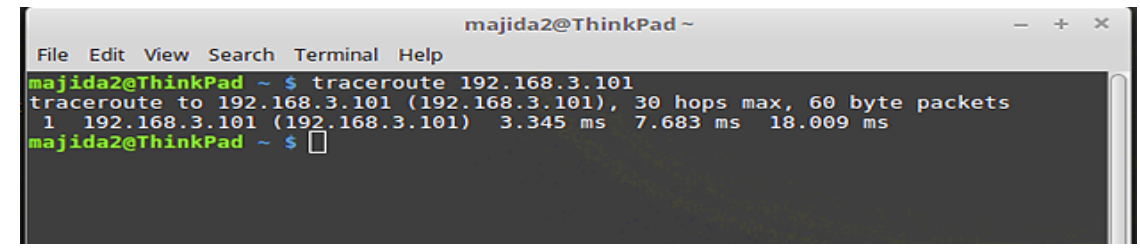

Figure 4. One-hop traceroute command execution

Figure 5 showed the result of two hops traceroute command, where node- 2 is used to forward the multimedia data to the destination node over multihop path.

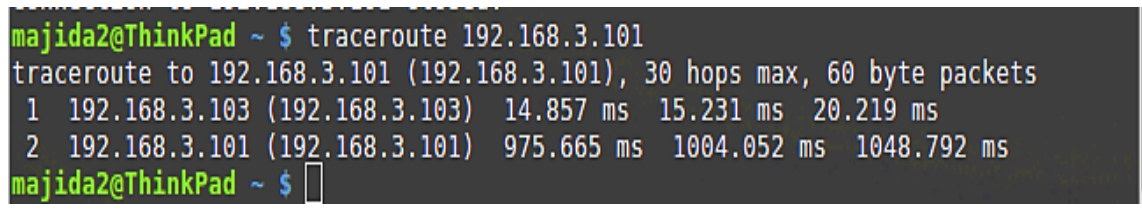

Figure 5. Two-hop traceroute command execution 


\section{Simulation setup}

Network Simulator (ns-3) is a discrete-event simulator, i.e. this simulator keeps tracking and scheduling a number of events to be executed at a particular simulation time, (i.e. the scheduled events are executed sequentially in time order) [12]. In this paper the system was evaluated for two scenarios.

Firstly, one-monitor received video from three-sources, as shown in Figure 6, where each of the threeQuadcopters equipped with a camera that recorded a video and sent it to the Monitor-node over the multi-hop network, which was represented by N-Rpi3. The first design leads to some problems at the destination node (congestion and interference), these problems were reduced in the second scenario.

Secondly, three-monitors received a video stream from three-sources, where each source was sent to a specific monitor to reduce the early mentioned network problems in the first scenario.

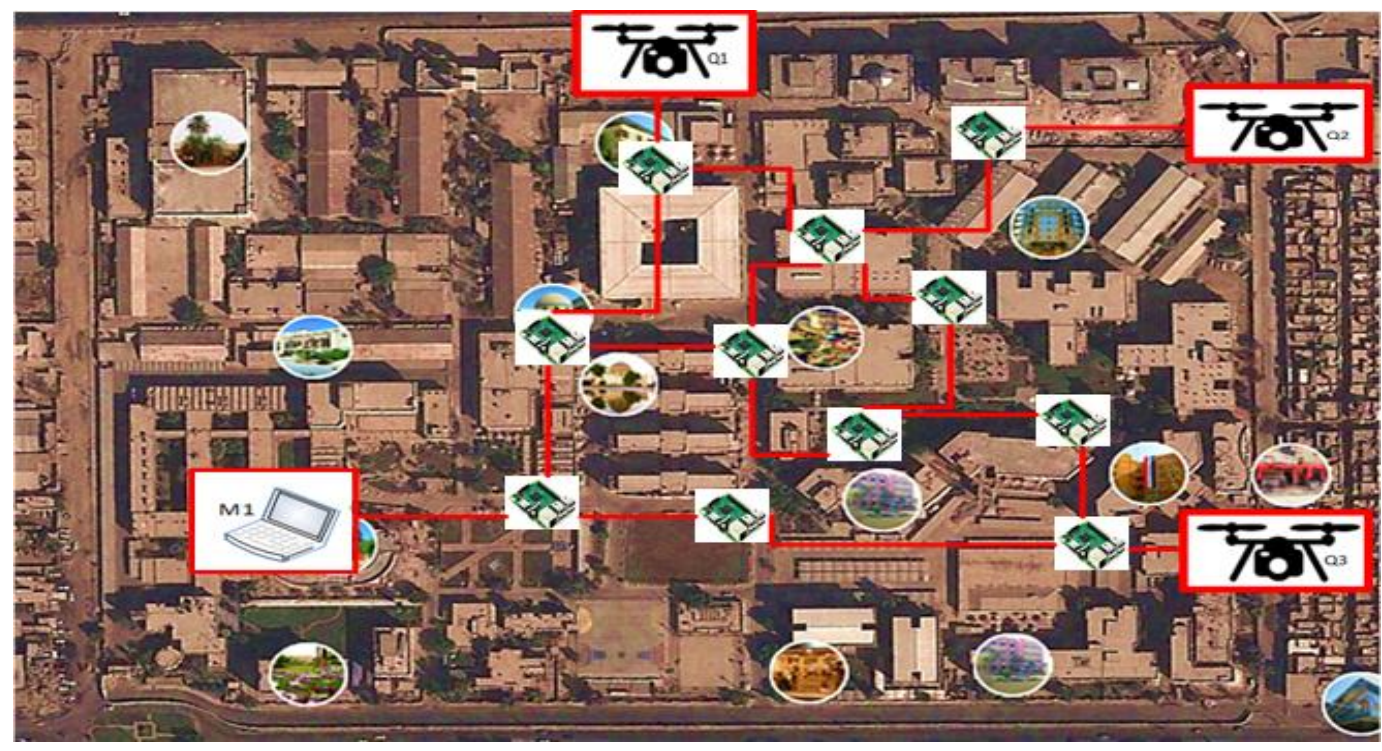

Figure 6. First scenario system design, Google Earth on 10/12/2018

In the simulator environment, a video trace file was used because it does not require any copyright, free and easy to be used by researchers.

The simulation platform in this paper was set to specific simulation parameters as shown in Table 1 for the first scenario, and Table 2 for the second scenario where all parameters were same except for the number of Monitor nodes being three instead of one.

Table 1. Simulation Setup Parameters-First Scenario

\begin{tabular}{ll}
\hline Parameters & Description \\
\hline Mobility model & Random Waypoint Mobility (RWM) \\
Simulation Area & $1050 * 780 \mathrm{~m}^{2}$ (University of Technology \\
& Campus) \\
Simulation Time & 250 Simulation sec. \\
Traffic Type & Variable Bit Rate (VBR) \\
Packet Size & 160 bytes \\
Node's Position Allocator & - Monitor-node with fixed position \\
& - M1, M2, M3 with Box Position \\
& Allocator (three-dimension mobility) \\
& - M4 is an n-nodes with Rectangle \\
& Position Allocator (two-dimension \\
Number of Nodes & mobility) \\
Set Seed & $30-120$ in steps of 30 \\
\hline
\end{tabular}

Table (2) Simulation Setup Parameters-Second Scenario

Parameters Description 


\begin{tabular}{ll}
\hline Node's Position Allocator & - Three-Monitor-nodes with a fixed position, \\
& nodes $(0,1$ and 2$)$ \\
- M1, M2, M3 with Box Position Allocator, \\
nodes $(3,4$ and 5), (three-dimension \\
mobility) \\
- M4 is an n-nodes with Rectangle Position \\
Allocator (two-dimension mobility) \\
\hline
\end{tabular}

The prototype of the proposed system was designed, implemented and tested using test-bed method, that system was constructed from three nodes connected using an ad-hoc network mechanism with OLSR as the routing protocol, (two Raspberry pi 3(Rpi3). One of them was mounted on a Quadcopter and was equipped with pi-camera v2 to record and send a real-time video stream to the monitor node that was represented by a Linux-PC, the other Rpi3 had been used to forward the data from the Quadcopters to the monitor over multihop path).

To evaluate the performance of the proposed prototype system with other routing protocols and different scenarios, as varying the number of nodes NS-3 simulator was used to estimate the optimum number of nodes and more efficient routing protocol to send the video stream from sources to monitor for the early mentioned scenarios.

The simulation was configured with different node's position allocators as mentioned in Tables (1) and (2), the details of the simulation's position allocator is as listed below:

i. Monitor/s, with fixed position allocator at thesimulation area.

ii. M1, M2, and M3 are set with box position allocator at three different positions of simulation area with a limited moving area of $(\mathrm{x}=25, \mathrm{y}=25, \mathrm{z}=10)$.

iii. M4 represents the number of rectangle position allocator nodes that forward data form (M1, M2, and M3) to monitor/s over the multi-hop path.

\section{Simulation results and discussion}

The results obtained from the two-scenarios were taken for different evaluation metrics that are; (delay, jitter, packet loss ratio (PLR) and packet delivery factor (PDF)) versus a variable number of nodes showed the difference in the performance of the two tested algorithms in the two study cases:

i. The results of the first case (one-monitor and three-sources).

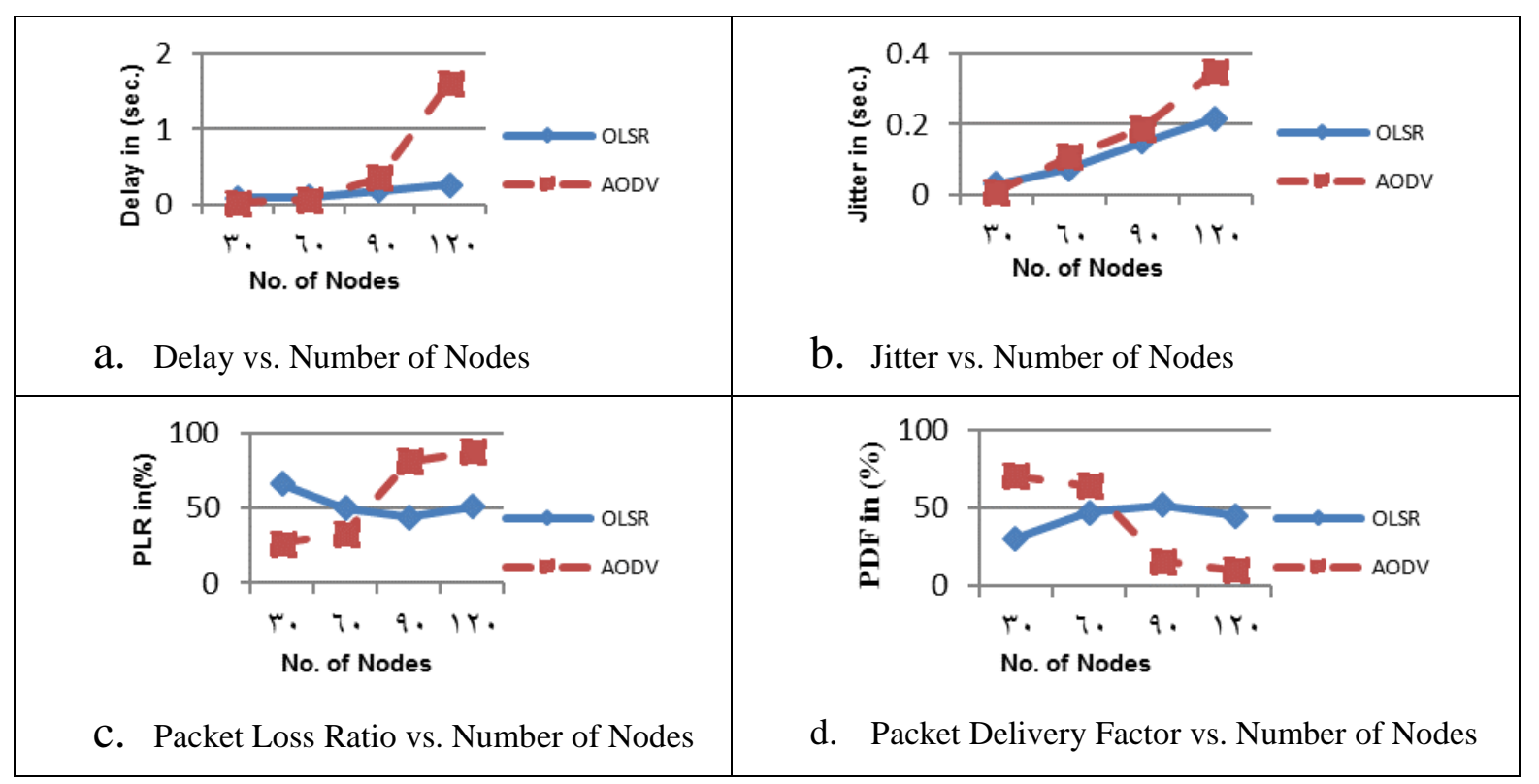

Figure 7. Performance evaluation curves for first scenario: a) Delay vs. number of nodes, b) Jitter vs. number of nodes, c) Packet loss ratio vs. mumber of nodes, and d). Packet delivery ratio vs. number of nodes 
The results shown in Figure 7 were comparing the performance of the two selected algorithms (AODV, OLSR) versus the number of nodes. The result of the comparison showed that; in (a) OLSR protocol had produced less delay when compared with AODV where the delay value exceeded $1.5 \mathrm{sec}$ when the number of nodes increased up to 120 node, the jitter had the same response in (b) as in (a), the packet loss ratio for OLSR in (c) decreased below 60\% as the number of nodes increased while for AODV the PLR increased sharply, finally in (d) the packet delivery factor of OLSR was more stable than AODV which reduced sharply when number of nodes exceeded 80 nodes while OLSR kept a PDF between $40 \%$ to $50 \%$.

ii. The curves are shown in Figure (8) to represent the performance of the system after modifying it to use three monitors instead of one:

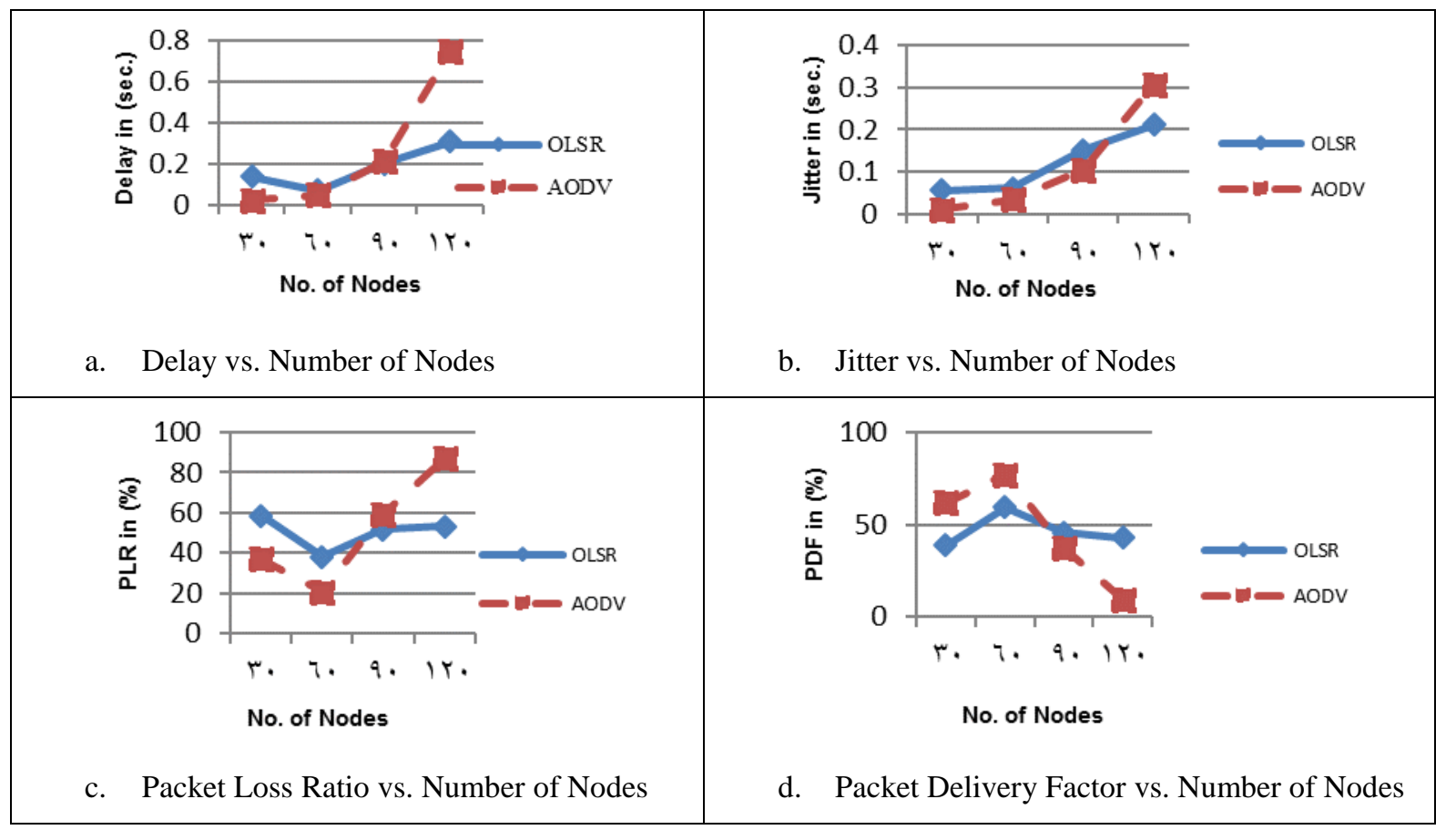

Figure 8. Performance evaluation curves for second scenario: a) Delay vs. number of nodes, b) jitter vs. number of nodes, c) packet loss ratio vs. number of nodes, and d) packet delivery ratio vs. number of nodes

The curves shown in Figure 8 showed that the delay of OLSR in (a) did not increase over 0.3 sec. while for AODV, the delay had exceeded $0.7 \mathrm{sec}$. When the number of nodes reached 120 nodes, the jitter vs. number of nodes in (b) increased after 90 nodes rapidly to reach up to $0.2 \mathrm{sec}$. For OLSR and $0.3 \mathrm{sec}$. for AODV, in (c) it's clear that the PLR for OLSR was stable at $50 \%$ for number of nodes over 90 nodes but for AODV, it reached $90 \%$ at 120 node. Finally in (d) the PDF had dropped pointedly when the number of nodes became over 90 nodes to be (40-50\%) for OLSR and below 10\% for AODV at 120 nodes.

\section{Conclusion}

In this paper, an aerial monitoring system was designed using Raspberry pi 3, ad-hoc model and Quadcopter, and was evaluated using test-bed method to test the configuration parameters of the ad-hoc mode and the performance of the OLSR protocol. The obtained results had shown that it performed very well in finding and establishing the path between two communicating nodes over one and two hops path. The proposed prototype system then was simulated using NS-3 to evaluate its performance for different scenarios using two protocols that are (AODV and OLSR), with variable performance parameters (Delay, Jitter, Packet Loss Ratio, and Packet Delivery Factor).

The results were taken for two scenarios: one-monitor receiving video from three-sources; and second scenario uses three-monitors receiving video stream from three-sources. The obtained results illustrated that the performance of OLSR protocol outperforms AODV in dense networks in both implemented scenarios and 
the results had shown that the optimum number of nodes needed to cover the simulation area with acceptable results of delay, jitter, PLR, and PDF is (80-90) nodes.

\section{References}

[1] Perkins, et al., “Ad hoc On-Demand Distance Vector (AODV) Routing/rfc 3561", 2003.

[2] T. Clausen and P. Jacquet, “Optimized Link State Routing Protocol /rfc 3626”, 2003.

[3] E. Yanmaz, S. Yahyanejad, B. Rinner, H. Hellwagner, and C. Bettstetter, "Drone networks: Communications, coordination, and sensing", Ad Hoc Networks, Institute of Information Technology, Austria, September 2018.

[4] C. Cambra, S. Sendra, J. Lloret, and L. Parra, "Ad hoc Network for Emergency Rescue System based on Unmanned Aerial Vehicles", Network Protocols and Algorithms, vol. 7, no. 4, p. 72, 2016.

[5] I. Al Barazanchi, "An Analysis of the Requirements for Efficient Protocols in WBAN," J. Telecommun. Electron. Comput. Eng., vol. 6, no. July, p. 43, 2015.

[6] Y. Gu, S. Fu, Y. Wan, and H. Zhang, "Drone-carried Ad Hoc WiFi system for Use in Fighting Wildfires", Department of Electrical Engineering, University of North Texas, 2003.

[7] O. Shrit, S. Martin, K. Al Agha, and G. Pujolle, "A new approach to realize drone swarm using ad-hoc networks", Conference Paper, Paris-Saclay University, France, IEEE, https://hal.archives-ouvertes.fr/hal$\underline{01696735}, 2017$.

[8] H. Ullah, M. Abu-Tair, S. Mcclean, P. Nixon, G. Parr, and C. Luo, "An Unmanned Aerial Vehicle Based Wireless Network for Bridging Communication", Conference Paper, 11th International Conference on Frontier of Computer Science and Technology, June 2017.

[9] D. Radu, A. Cretu, B. Parrein, J. Yi, C. Avram, and A. Astilen, "Flying Ad Hoc Network for Emergency connected to a Fog System", Advances in Internet, Data \&amp; Web Technologies (Vol. 17). Cham: Springer International Publishing. https://Doi.org/10.1007/978-3-319-75928-9, February 2018.

[10] I. Al Barazanchi, H. R. Abdulshaheed, S. A. Shawkat, and S. R. Binti, "Identification key scheme to enhance network performance in wireless body area network," Period. Eng. Nat. Sci., vol. 7, no. 2, pp. 895-906, 2019.

[11] “University of Technology General Information,” 2011.

[12] NS-3 Official Site/ https://www.nsnam.n 